\title{
MYXOME DU MAXILLAIRE A PROPOS D'UN CAS
}

\author{
I. GASSAB, A. HAMROUN, K. HARRATHI, F. BEN MAHMOUD, S. KADHI, F. EL KADHI, \\ CH. HAFSA*, J. KOUBAA, A.GASSAB \\ SERVICE ORL. \\ *SERVICE DE RADIOLOGIE. \\ HÔPITAL FATTOUMA BOURGUIBA MONASTIR
}

\begin{abstract}
RESUME
Les myxomes des maxillaires sont des tumeurs bénignes rares dont la pathogénie reste encore controversée. Leur indolence et la pauvreté des manifestations qui les accompagnent rendent leur diagnostic souvent tardif et leur prise en charge difficile. Nous présentons un cas de myxome du maxillaire chez un nourrisson âgé de 12 mois suivi d'une revue de la littérature permettant de synthétiser les données cliniques ainsi que la stratégie thérapeutique à adopter.
\end{abstract}

Mots clés : Myxomes - os maxillaire - tumeur bénigne.

\section{SUMMARY}

Maxillary myxoma are rare benign tumours whose pathogenesis remains extremely discussed. The poor clinical picture and the absence of pain makes the diagnosis difficult. We report a pediatric case of maxillary myxoma of a 12- monthold infant and review the clinical features, radiographic evaluation and the appropriate treatment

Key words : Myxoma - maxillary - benign tumor.

\section{INTRODUCTION}

Les myxomes des maxillaires sont des tumeurs rares, survenant le plus souvent chez l'adulte jeune. Ces tumeurs dont la pathogénie est encore controversée représentent 0,04 à $0,6 \%$ des tumeurs de la cavité buccale et 3 à $7 \%$ des tumeurs odontogéniques [1, 2, 3].

Les séries rapportées dans la littérature portent sur des cas isolés [1, 2, 4, 5, 6, 7, 8, 9, 10,11]. Bien que bénignes, ces tumeurs sont localement agressives imposant une prise en charge appropriée. Leur traitement est chirurgical.

Nous rapportons le cas d'un myxome du maxillaire chez un nourrisson âgé de 12 mois. A cette occasion, les auteurs rappellent les caractéristiques cliniques, radiologiques et évolutives tout en insistant sur la prise en charge de cette pathologie.

\section{OBSERVATION}

Un nourrisson âgé de 12 mois de sexe féminin, sans antécédent particulier, a été amené par ses parents pour voussure de la région maxillaire droite apparue depuis un mois de façon isolée. L'examen de la face a mis en évidence une tuméfaction indolore, faisant $5 \mathrm{~cm}$ de grand axe, effaçant le sillon naso-génien droit (fig 1). La rhinoscopie antérieure a trouvé une réduction de la perméabilité de la fosse nasale droite par un refoulement de la cloison inter sinusonasale. La fosse nasale gauche était perméable et le cavum était libre. L'ouverture buccale était normale, il n'existait ni voussure du palais ni lésion gingivale ni adénopathie cervicale. L'examen ophtalmologique, neurologique et somatique était normal.

Le scanner du massif facial a objectivé un volumineux processus expansif polycyclique du maxillaire érodant les parois osseuses (fig2). La lésion a détruit également le plancher orbitaire sans envahissement intra orbitaire ; la fosse infra-temporable droite est non envahie.

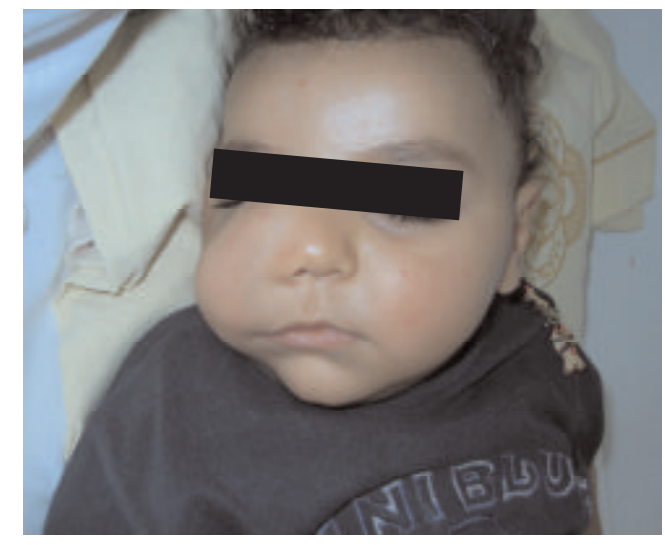

Fig. 1 : Photo pré-opératoire d'un enfant de 12 mois présentant une importante déformation faciale par la tumeur du maxillaire qui efface le sillon naso-génien droit.

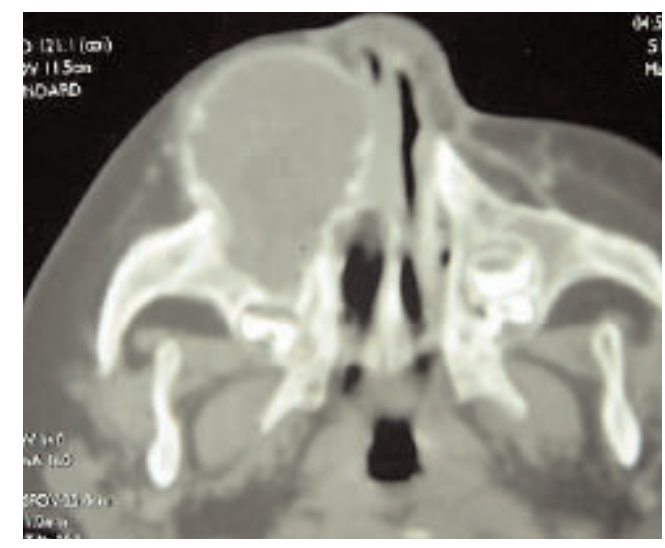

Fig. 2 : TDM du massif facial en coupe axial : Volumineux processus expansif uniloculaire paranasale droit, saillant, développé au niveau du maxillaire droit. 
L'enfant a été opéré sous anesthésie générale, par un abord para-latéro-nasal droit (fig3). L'aspect per-opératoire était celui d'une tumeur tissulaire prenant le maxillaire droit érodant l'arcade zygomatique et le plancher de l'orbite. La tumeur est réséquée en totalité en monobloc (fig4 ,5). La fermeture du site opératoire était possible sans reconstruction du défect osseux.

L'examen anatomo-pathologique de la pièce opératoire a retenu le diagnostic de myxome du maxillaire. Les suites opératoires étaient simples. L'enfant est suivi régulièrement depuis deux ans. La tumeur n'a pas récidivé.

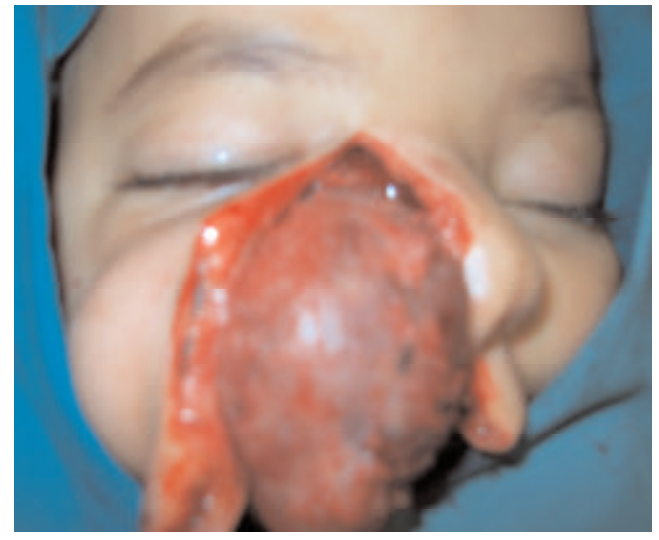

Fig. 3 : Vue per-opératoire montrant l'abord para-latéro-nasal droit de la tumeur.

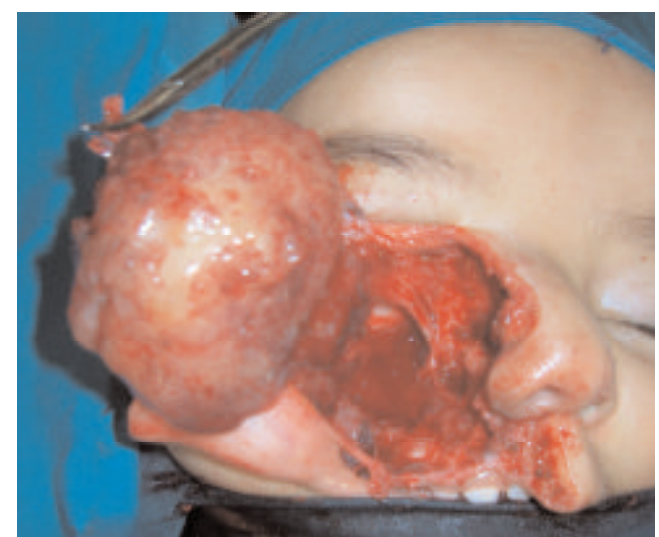

Fig. 4 : Vue per-opératoire : Exérèse complète de la tumeur en monobloc.

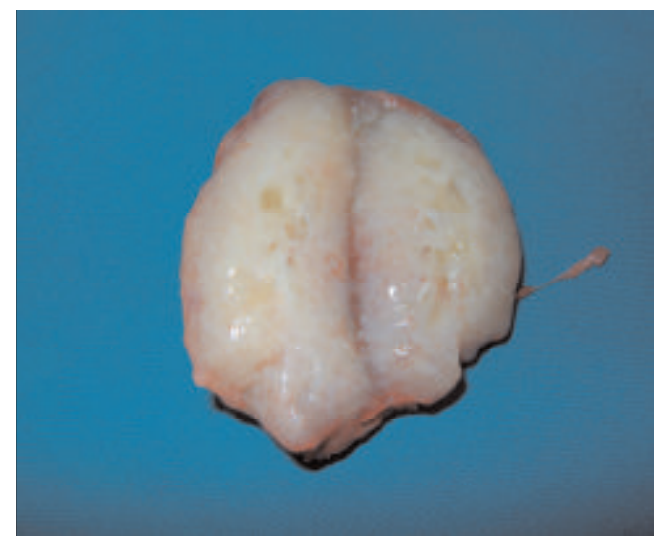

Fig. 5 : Pièce opératoire

\section{DISCUSSION}

Les myxomes des maxillaires sont des tumeurs bénignes rares dont la pathogénie reste indéterminée [1] Deux théories se partagent la pathogénie de cette tumeur. La première plaide en faveur d'un développement tumoral aux dépens de cellules souches persistant au sein du tissu conjonctif. La deuxième plaide en faveur d'une dégénérescence myxomateuse du stroma fibreux [12, 13]. La présence occasionnelle d'un épithélium odontogénique au sein de la tumeur suppose une origine dentaire [12]. Des facteurs de prédisposition ont été évoqués, notamment le syndrome de Carney. Ce syndrome héréditaire à transmission autosomique dominante, associe une hyperactivité endocrine, des lésions cutanées lentigineuses et des myxome multiples [14].

Ces tumeurs touchent essentiellement l'adolescent, rarement l'enfant et l'adulte [2, 3, 4]. Elles peuvent apparaître également chez le nourrisson dés les premiers mois de la vie $[8,9,10]$. Notre patiente est âgée de 12 mois.

Au niveau cervico-facial, deux formes sont décrites : le myxome osseux, il dérive de la mandibule, du maxillaire, de l'os nasal rarement du rocher ; quant au myxome des tissus mous, il dérive des tissus mous para-pharyngés, de la glande parotide et du larynx.

Le myxomes osseux touche plus la mandibule que le maxillaire [2]. Le myxome du maxillaire pourrait naître du mésenchyme odontogène, théorie étayée notamment par l'existence des dents incluses associées ; il pourrait également dériver des tissus non-odontogènes.

Les manifestations cliniques sont tardives. Les malades consultent le plus souvent pour une déformation du massif facial pouvant atteindre des volumes monstrueux [8]. Le scanner, examen non spécifique, permet d'étudier la topographie de la tumeur [6]. Cette dernière peut prendre l'aspect de lésions kystiques solitaires ou multiples, formées par des loci entourés de matériel osseux [2, 15, 16]. Ces lésions ostéolytiques sont souvent entourées par une zone marginale de haute densité radiographique $[2,6,16]$. Elle peut aussi se manifester sous forme de spicule résultant de l'effraction tumorale à travers la corticale osseuse envahissant les tissus mous avoisinants [4, 6, 16]. Cet aspect impose un diagnostic différentiel avec l'ostéosarcome et les autres tumeurs odontogéniques. Les myxomes ont un signal variable à I' IRM, souvent hétérogène. Un hyposignal en T1 et hypersinal T2 est très évocateur mais inconstant. Le rehaussement après injection de produit de contraste est variable $[6,15,16]$.

L'aspect anatomopathologique de ces tumeurs est caractérisé par la présence des cellules rondes et anguleuses disséminées dans un abondant stroma mucoïde contenant des muccopolysaccharides et un réseau de fibres réticulaires [5, 7]. II n'y a aucune atypie et l'activité mitotique est faible. Un épithélium odontogénique retrouvé au sein la tumeur plaide en faveur de l'origine dentaire pour certains auteurs. Le diagnostic différentiel se pose avec 
les tumeurs dérivées des cellules de Schwann, le liposarcome myxoïde, le rhabdomyosarcome ou encore les tumeurs fibroblastiques ou chondroïdes [5].

Bien que bénignes, ces tumeurs sont localement agressives avec un potentiel lytique et invasif important $[5,8$, 14]. Des formes avec envahissement endocrâniennes et atteinte des paires crâniennes sont décrites [14]. Leur croissance peut être rapide due à une accumulation de substances mucoïdes.

Le traitement de ces tumeurs est chirurgical. La voie d'abord dépend du siège de la tumeur et de son extension. L'énucléation et le simple curetage sont plus pourvoyeurs de récidives, ces dernières sont plus fréquentes pour les myxomes du maxillaire que mandibulaire $(31,2 \%$ de récidive mandibulaire, $80,9 \%$ de récidive maxillaire).

Pour les grosses tumeurs, il est souvent nécessaire de pratiquer une plastie par des greffons osseux, des lambeaux régionaux de rotation ou des lambeaux à distance $[5,7,11]$.

Un long suivi clinique et radiologique s'impose afin de diagnostiquer d'éventuelles récidives

\section{CONCLUSION}

Les myxomes du maxillaire sont des tumeurs bénignes, rares, de croissance lente, potentiellement agressives. Ils surviennent préférentiellement chez l'adolescent rarement chez l'enfant. Le scanner, souvent complété par I'IRM, apporte des informations importantes sur l'extension et l'envahissement tumoral. Le diagnostic repose aussi sur l'examen anatomopathologique. Le traitement est chirurgical. Le pronostic est bon.

\section{REFERENCES}

1- Wong G.B., Large odontogenic myxoma of the mandibule treated by sagittal ramus osteotomy and peripheral ostectomy, Journal of Oral and Maxillo-Facial Surgery 1992; 50: 1221-1224.

2- Dezotti MSG., Azevedo LR., Fontão F., Capelozza A., Sant'ana E. Odontogenic Myxoma A Case Report and Clinico-Radiographic Study of Seven Tumors. J Contemp Dent Pract 2006 ; 7 (1): 117-124.

3- Oluseyi F. Ajayi, Akinola L Ladeinde, Wasiu L Adeyemo, Mobolanle O Ogunlewe. Odontogenic tumors in Nigerian children and adolescents- a retrospective study of 92 cases World J Surg Oncol. 2004; 2(1): 39.

4- Heymans O., Nélissen X., Gilon Y., Jacquemin D., Fissette J. Myxome de la mandibule à propos d'un cas. Rev. Stomatol. Chir. maxillofac. 2002; 103(4) : 239-241.

5- Brian W. Rotenberga, Sam J. Daniela, lain A. Nishb, Bo Y. Nganc, Vito Fortea, Myxomatous lesions of the maxilla in children: a case series and review of management. Int. J. of Pediatric Otorhinolaryngology 2004; 68: 1251-1256.

6- Jae-Duk Kim, Kwang-Won Kim, Sung-Hoon Lim Odontogenic myxoma: a case report with recent image modalities. Korean J Oral Maxillofac Radiol 2004; 34 : 199-202.

7- Ög ütcen-Toller M., Sener I., Kasap V., Cakir-Özkan N. Maxillary Myxoma: Surgical Treatment and Reconstruction with Buccal Fat Pad Flap: A Case Report. J Contemp Dent Pract 2006 ; ( 7)1: 107-116.

8- Vikas Malhotra, Ashwani Sethi, Shilpi Malhotra, Deepika Sareen, Rajeev Puri: Massive Odontogenic Fibromyxoma Of Maxilla. The Internet Journal of Oncology. 2005; 3 (1):
9- Wachtera Bryan G., Steinbergb Mark J., Darrowc David H., McGinnc Jonathan D., Parka Albert H. Odontogenic myxoma of the maxilla: a report of two pediatric cases International Journal of Pediatric Otorhinolaryngology (2003) 67, 389-/393.

10- Sinead Fenton, Pieter J. Slootweg, Enwin A. Dunnebier Maarten Ph. Mourits Odontogenic Myxoma in a 17-Month-Old Child: A Case Report J Oral Maxillofac Surg $2003 ; 61: 734-736$.

11- Spencer K.R., Smith A., Odontogenic myxoma: Case report with reconstructive considerations Australian Dental Journal 1998; 43:(4): 000-000.

12- Ghosh BC., Huvos AG., Gerold FP., Miller TR. Myxoma of the jaw bones. Cancer, 1973; 31: 237-40.

13- Stout AP. Myxoma, the tumor of primitive mesenchyme. Ann Surg, 1948; 127: 706-19.

14- Zeineb O., Rim L., Slim T., Said G., Ahmed E., Amor G., Abderrahmen L.Myxome du maxillaire. JFORL $2003 ; 52(4): 171-174$.

15-. Aquilino R N., Tuji F M., Eid NL.M, Molina O F., Joo H Y., Neto F H. Odontogenic myxoma in the maxilla: A case report and characteristics on CT and MR. Oral Oncology Extra (2006) ; 42(4) : 133-136.

16- Asaumi J., Konouchi H., Hisatomi M., Kishi K. Odontogenic myxoma of maxillary sinus: CT and MR-pathologic correlation. European Journal of Radiology $2001 ; 37: 1-4$. 